\title{
Arranjos para efeitos fixos e estruturas de (co)variâncias residuais para análises de medidas repetidas do peso de bovinos da raça Canchim ${ }^{1}$
}

\author{
Fábio Luiz Buranelo Toral' ${ }^{2}$, Maurício Mello de Alencar ${ }^{3}$, Alfredo Ribeiro de Freitas ${ }^{3}$
}

\footnotetext{
1 Parte da dissertação de Mestrado do primeiro autor apresentada ao Programa de Pós-Graduação em Genética e Melhoramento Animal da Universidade Estadual Paulista / Faculdade de Ciências Agrárias e Veterinárias de Jaboticabal. Projeto financiado pela Embrapa e FAPESP.

2 Universidade Federal de Mato Grosso.

${ }^{3}$ Embrapa Pecuária Sudeste. Bolsista do CNPq.
}

RESUMO - Este trabalho foi realizado com o objetivo de selecionar o arranjo para efeitos fixos e a estrutura de (co)variância residual que melhor representam a variabilidade dos pesos dentro do rebanho e dentro dos indivíduos, considerando-se dados de pesos de bovinos analisados como medidas repetidas. Foram utilizados dados de peso de 3.690 bovinos Canchim, obtidos ao nascimento, à desmama, aos 12 e aos 18 meses de idade. Analisaram-se diferentes arranjos para os efeitos fixos (grupos de contemporâneos e/ou efeitos principais de ano, mês ou época de nascimento e sexo do bezerro) e diferentes estruturas de (co)variâncias para os resíduos, considerando-se ou não alteração da variância residual ao longo da vida do animal e alteração da correlação entre as medidas tomadas em intervalos diferentes. Os resultados indicaram que o arranjo mais adequado dos efeitos fixos para representar a variabilidade dos pesos dos animais dentro do rebanho foi o grupo de contemporâneos formado por ano, mês e sexo do bezerro e que as melhores estruturas de (co)variâncias residuais foram a Fator Analítico de Primeira Ordem e a Não Estruturada, que consideram o aumento das variâncias com o aumento da idade do indivíduo e as correlações diferentes para cada par de medidas de peso.

Palavras-chave: dados longitudinais, efeitos principais, grupos de contemporâneos, resíduos correlacionados

\section{Fixed effects arrays and residual covariance structures to analyze sequential weights of Canchim beef cattle}

\begin{abstract}
The aim of this work was to evaluate arrays of fixed effects and residual covariance structures that best fit the herd and the animal variability to weights at birth, weaning, twelve and eighteen months of 3,690 Canchim animals. Different arrays of fixed effects (contemporary groups and, or the main effects of year, month or season of birth and sex) and different residual covariance structures (considering or not change of variance and of correlation between weights at different ages) were studied. The results indicated that the most adequate array of fixed effects to fit herd variability was the contemporary group of year, month and sex. The best residual covariance structures were the First-Order Factor Analytic and the Unstructured, which consider increase of residual variance as age increases and different correlation between pair of weights at different ages.
\end{abstract}

Key Words: contemporary groups, longitudinal data, main effects, residual correlation

\section{Introdução}

Vários estudos têm sido conduzidos com o objetivo de investigar a resposta de uma característica ao longo do tempo. Assim, as medidas repetidas são obtidas nos mesmos indivíduos, em tempos diferentes, e, possivelmente, sob diferentes condições experimentais. Nesses casos, pode existir uma estrutura de (co)variância única para representar a variabilidade das medidas dentro dos indivíduos de uma população, mas os parâmetros dessa estrutura podem variar entre os indivíduos (Laird \& Ware, 1982).
Medidas repetidas de uma característica, como o peso em diferentes idades, avaliadas no mesmo animal, são correlacionadas entre si. A correlação e a variância dessas medidas podem variar ao longo do tempo e podem produzir uma estrutura de (co)variância complicada, a qual requer métodos estatísticos especiais para a análise (Littell et al., 1998).

Freitas et al. (2005) estudaram os pesos de bovinos das raças Nelore, Guzerá, Gir e Indubrasil sob o enfoque de medidas repetidas e sugeriram que o estudo dessas características considerando-se uma estrutura de (co)variância residual entre as medidas é mais eficiente que a análise 
univariada, normalmente feita por meio do procedimento GLM do SAS, a qual considera as medidas repetidas como subparcelas. Gröhn et al. (1999) estudaram o efeito da cetose sobre a produção de leite em vacas da raça Holandesa considerando a produção total de leite ajustada para 305 dias de lactação e considerando os controles de produção mensais como medidas repetidas, com estruturas de (co)variâncias residuais entre as medidas. Os autores concluíram que, por meio do segundo tipo de análise, foi possível identificar diminuição significativa da produção de leite em vacas com cetose, o que não ocorreu com a primeira análise, o que evidenciou maior eficiência da análise de medidas repetidas, em relação à análise univariada, para detectar efeitos significativos das fontes de variação.

A escolha da melhor estrutura de (co)variância residual e do modelo mais adequado é fundamental na análise de dados de medidas repetidas, caso contrário, os resultados podem ocasionar conclusões equivocadas. Segundo Littell et al. (1998), a escolha da estrutura de (co)variância para os resíduos é importante porque os erros-padrão das médias são dependentes desta escolha e, caso se opte por uma estrutura inadequada, os erros-padrão podem ser viesados.

O procedimento MIXED do SAS (SAS, 2000) disponibiliza ao usuário em torno de 40 tipos de estruturas de (co)variâncias residuais e critérios para escolha da melhor estrutura para determinado conjunto de dados. Esses critérios, em geral, incluem o valor da função de máxima verossimilhança restrita, o número de dados analisados e de parâmetros a serem estimados.

Para determinado conjunto de dados de pesos de bovinos analisados como medidas repetidas, a estrutura de (co)variância pode variar entre as diversas raças ou grupos genéticos. Freitas et al. (2005), analisando dados de nove pesagens, realizadas em intervalos trimensais do nascimento aos dois anos de idade, concluíram que a estrutura Fator Analítico de Primeira Ordem (FA(1)) foi a mais adequada para as raças Nelore e Indubrasil, enquanto a Não Estruturada (UN) foi a mais adequada para as raças Guzerá e Gir. Todavia, as estruturas que requerem a estimação de maior número de parâmetros tendem a proporcionar os melhores ajustes (Gröhn et al., 1999; Freitas et al., 2005; Lorenzo-Bermejo et al., 2003).

Este trabalho foi realizado com o objetivo de selecionar o arranjo para efeitos fixos e a estrutura de (co)variância residual que melhor representem a variabilidade dentro do rebanho e dentro dos indivíduos, utilizando-se dados de medidas repetidas do peso de bovinos da raça Canchim.

\section{Material e Métodos}

Os dados utilizados neste estudo foram provenientes do controle de desenvolvimento ponderal de bovinos da raça Canchim, do rebanho da Embrapa Pecuária Sudeste, localizada em São Carlos, SP. Foram analisados os dados de 3.690 animais nascidos entre os anos de 1956 e 2000 (exceto 1957 e 1999), pesados ao nascimento (PN), à desmama (P240), aos 12 (P365) e aos 18 meses de idade (P550). As idades dos animais no momento das pesagens à desmama, aos 12 e aos 18 meses de idade variaram de 180 a 300, de 305 a 405 e de 490 a 610 dias de idade e os pesos utilizados foram padronizados para as idades de 240,365 e 550 dias, respectivamente, considerando-se o ganho de peso diário de cada animal durante o período que antecedeu cada pesagem. Para análise, foram utilizados os dados de pesos cujas classes de efeitos fixos formadas pelo ano, mês de nascimento e sexo do animal possuíam pelo menos cinco observações. Os animais foram criados em regime exclusivo de pastagens e maiores informações sobre o manejo podem ser obtidas em Mello et al. (2002).

Inicialmente, os dados foram submetidos a análises exploratórias com o objetivo de diagnosticar a qualidade. De acordo com Freitas (2003), dados de campo, principalmente em virtude de problemas de coleta, apresentam "outliers", correlações absurdas, afastamento da distribuição normal, heterogeneidade de variâncias dentro das classes de efeitos fixos e grau acentuado de assimetria e curtose, anomalias que podem omitir resultados importantes quando se utilizam procedimentos tradicionais de análises.

Os coeficientes de assimetria e curtose, as (co)variâncias e as correlações fenotípicas para os pesos observados foram calculados sem qualquer ajuste para as fontes de variação com o propósito de compará-los àqueles obtidos nas análises utilizando-se modelos mais parametrizados. A assimetria é uma forma de a distribuição dos dados ser mais viesada em uma direção que em outra. Quando se considera uma distribuição simétrica como a normal, a curtose é uma medida do grau de achatamento dessa distribuição (SAS, 2000). Ambas são importantes nas análises exploratórias dos dados (Freitas, 2003).

Posteriormente, os dados de pesos, medidos nas diferentes idades do animal, foram considerados medidas repetidas e analisados pelo seguinte modelo linear misto:

$$
y_{i l j v}=\mu+a_{i l v}+f_{l}+i d_{j}+b_{1}\left(i v_{v}-i \bar{v}\right)+b_{2}\left(i v_{v}-i \bar{v}\right)^{2}+e_{i j j v} \text {, }
$$
em que $y_{i l j}$ é a resposta do animal $i$, submetido aos efeitos fixos $l$, na idade $j$, filho da vaca com idade $v ; \mu$, a constante geral; $a_{i v}$, o efeito aleatório do animal $i$ submetido 
aos efeitos fixos $l$, filho da vaca com idade $v ; f_{l}$, o efeito do arranjo de efeitos fixos; $i d_{j}$, o efeito da idade $j(j=0,240$, 365 e 550 dias); $b_{1}$ e $b_{2}$, os coeficientes linear e quadrático, respectivamente, do efeito da idade $v$, da mãe do animal $i$; $i v$ e $i \bar{v}$, a idade da vaca e a média de idade das vacas; e $e_{i l j v}$, o erro aleatório associado a cada observação.

Sob a forma matricial, o modelo pode ser representado por:

$$
y=X b+Z a+e
$$

em que $y=$ vetor de observações $(14.760 \times 1) ; X=$ matriz de incidência dos efeitos fixos (14.760 x número de efeitos fixos, que varia em função do arranjo de efeitos fixos considerado); $b=$ vetor das soluções para os efeitos fixos (número de efeitos fixos x 1); $Z$ = matriz de incidência dos efeitos aleatórios $(14.760 \times 3.690)$; $a=$ vetor de soluções dos efeitos aleatórios $(3.690 \times 1) ; e=$ vetor de resíduos (14.760 x 1).

As pressuposições assumidas são:

$$
\left[\begin{array}{c}
y \\
a \\
e
\end{array}\right] \sim N M V\left\{\left[\begin{array}{c}
X b \\
0 \\
0
\end{array}\right] ;\left[\begin{array}{ccc}
Z G Z^{\prime}+R & Z G & R \\
G Z^{\prime} & G & 0 \\
R & 0 & R
\end{array}\right]\right\},
$$

em que $G=I \sigma_{a}^{2}$, sendo $I$ uma matriz identidade de ordem 3.690 e $\sigma_{a}^{2}$ a variância genética aditiva direta; $\mathrm{R}$, uma matriz bloco-diagonal definida por $C \otimes I \sigma_{e}^{2}$, em que $C$ é uma matriz de (co)variâncias entre as medidas de cada animal, de dimensão quatro; $I$, uma matriz identidade de ordem 3.690; $\sigma_{e}^{2}$, a variância residual; e $\otimes$, o produto direto de Kronecker. Os efeitos fixos foram considerados como efeitos principais e/oucomo grupos de contemporâneos, analisando-se seis arranjos: um (AEF1) envolvendo os efeitos principais de ano e mês de nascimento e sexo do bezerro; dois (AEF2), os efeitos principais de ano e trimestre de nascimento e sexo do bezerro; três (AEF3), o grupo de contemporâneos composto por ano e mês de nascimento e o efeito principal de sexo do bezerro; quatro (AEF4), o grupo de contemporâneos composto por ano e trimestre de nascimento e o efeito principal de sexo do bezerro; cinco (AEF5), o grupo de contemporâneos composto por ano, mês de nascimento e sexo do bezerro; e seis (AEF6), o grupo de contemporâneos composto por ano, trimestre de nascimento e sexo do bezerro.

Estes arranjos foram estudados em conjunto com as estruturas de (co)variâncias (Tabela 1) utilizadas por Bozdogan (1987), Wolfinger (1993), Vonesh et al. (1996), Littell et al. (1998), Gröhn et al. (1999), Lorenzo-Bermejo et al. (2003) e Freitas et al. (2005).

As estruturas $\operatorname{AR}(1)$ : auto-regressiva de primeira ordem; $\operatorname{ARMA}(1,1)$ : auto-regressiva de primeira ordem com média móvel; CS: simetria composta; TOEP: Toeplitz; TOEP(2): Toeplitz com duas bandas; e VC: componentes de variância consideram homogeneidade de variâncias, enquanto as estruturas ARH(1): auto-regressiva heterogênea de primeira ordem; $\mathrm{CSH}$ : simetria composta heterogênea; FA(1): fator analítico de primeira ordem; HF: Huynh Feldt; TOEPH: Toeplitz heterogênea; e UN: não-estruturada, consideram heterogeneidade de variâncias. $\mathrm{Na}$ estrutura CS, todas as covariâncias são iguais e, na VC, as covariâncias são nulas. As demais estruturas consideram diferentes covariâncias para cada par de medidas.

Para escolha do arranjo para efeitos fixos e da estrutura de (co)variância que melhor representam a variabilidade entre e dentro de animais, respectivamente, foram utilizados os critérios "-2 Residual Log Likelihood" (-2RLL) e "Consistent Akaike's Information Criterion" (CAIC), obtidos com as seguintes fórmulas (SAS, 2000; Wolfinger, 1993):

$$
\begin{gathered}
-2 R L L=(n-p) \ln (2 \pi)+\ln \left|Z G Z^{\prime}+R\right|+\ln \left|X^{\prime}\left(Z G Z^{\prime}+R\right)^{-1} X\right|+ \\
(y-X b)^{\prime}\left(Z G Z^{\prime}+R\right)^{-1}(y-X b) \mathrm{e} \\
C A I C=-2 R L L+d(\ln k+1),
\end{gathered}
$$

em que $n=$ número de animais (3.690); $p=$ posto da matriz $\mathrm{X} ; k=$ número de observações utilizadas (14.760); e as demais matrizes e vetores como definidos anteriormente.

Os parâmetros das matrizes de (co)variâncias residuais foram estimados pelo método da Máxima Verossimilhança Restrita, observando-se que, no procedimento MIXED do SAS, o máximo é obtido pelo método de Newton-Raphson. Como critério de convergência do algoritmo de busca do ponto de máximo da função de verossimilhança restrita, adotou-se o padrão do procedimento MIXED do SAS: $\frac{g_{k}^{\prime} H_{k}^{-1} g_{k}}{\left|f_{k}\right|} \leq 1 * 10^{-8}$, em que $f_{k}=$ valor da função de interesse (verossimilhança restrita); $g_{k}=$ primeira derivada da função de interesse; e $H_{k}=$ segunda derivada da função de interesse, ambos na iteração $k$ (SAS, 2000).

$\mathrm{O}$ arranjo mais adequado para os efeitos fixos foi obtido por meio do teste de $\chi^{2}$ para os critérios -2RLL e CAIC, com graus de liberdade iguais à diferença entre os graus de liberdade dos resíduos dos modelos com diferentes arranjos para efeitos fixos. Na escolha da estrutura de (co)variância mais adequada, também foi realizado o teste de $\chi^{2}$ para os critérios de ajuste proporcionados pelo SAS, sendo o grau de liberdade do teste igual à diferença entre o número de parâmetros das estruturas em questão. Valores de -2RLL e de CAIC mais próximos de zero indicam melhor ajuste do modelo (SAS, 2000). 
Tabela 1 - Estruturas de (co)variâncias utilizadas nas análises ${ }^{1}$ Table 1 - Covariance structures used in the analyses ${ }^{1}$

Estruturas com homogeneidade de variâncias

Structures with homogeneous variances

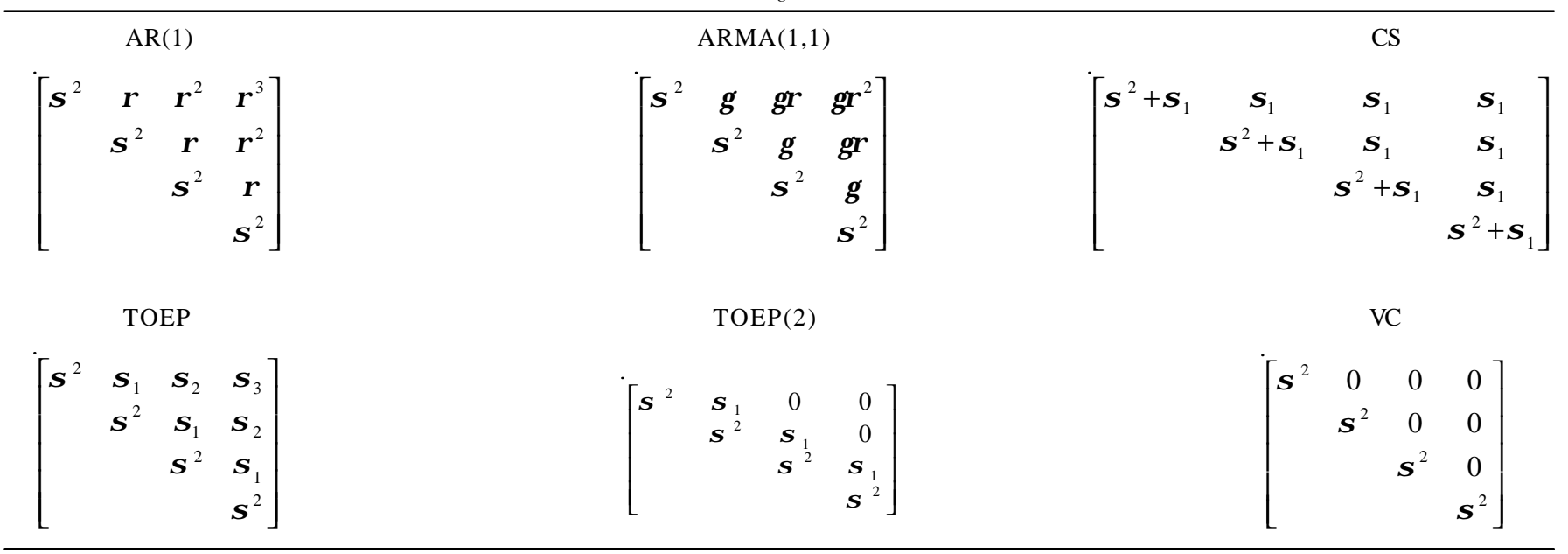

Estruturas com heterogeneidade de variâncias

Structures with heterogeneous variances

ARH(1)
$\left[\begin{array}{cccc}\sigma_{1}^{2} & \sigma_{1} \sigma_{2} \rho & \sigma_{1} \sigma_{3} \rho^{2} & \sigma_{1} \sigma_{4} \rho^{3} \\ & \sigma_{2}^{2} & \sigma_{2} \sigma_{3} \rho & \sigma_{2} \sigma_{4} \rho^{2} \\ & \sigma_{3}^{2} & \sigma_{3} \sigma_{4} \rho \\ & & \sigma_{4}^{2}\end{array}\right]$
HF

Após a escolha do melhor arranjo para efeitos fixos e da estrutura de (co)variância residual mais adequada, foram realizadas análises de variância unicaracterísticas para os pesos de cada idade. Os resíduos associados a cada pesagem obtidos nas análises com estruturas de (co)variância e nas unicaracterísticas foram utilizados para os cálculos dos coeficientes de assimetria e curtose.

\section{Resultados e Discussão}

Na Tabela 2 são apresentados as médias e os respectivos desvios-padrão, os valores mínimos e máximos e os coeficientes de assimetria e curtose e, na Tabela 3, são apresentadas as (co)variâncias e as correlações para PN, P240, P365 e P550.
As médias de pesos e os respectivos desvios-padrão para as diferentes idades mantiveram-se dentro do intervalo descrito na literatura para esta raça (Mascioli et al., 1996; ABCCAN-Embrapa-Geneplus, 2002), sugerindo que os resultados deste estudo podem ser generalizados para ou tros rebanhos da raça Canchim, enquanto os coeficientes de assimetria e curtose observados foram menores que os observados por Freitas (2003) para dados de campo de bovinos da raça Nelore provenientes de vários rebanhos mantidos em diferentes regiões do país. Essas diferenças podem ser atribuídas, em parte, ao fato de que os dados utilizados neste estudo foram provenientes de um rebanho experimental, enquanto aqueles utilizados por Freitas (2003) foram obtidos de rebanhos comerciais utilizando-se diferentes metodologias de pesagem. 
Tabela 2 - Médias $(\bar{x})$, desvios-padrão (s), valores mínimos (min) e máximos (max) e coeficientes de assimetria (ass) e curtose (cur) dos peșos ao nascimento (PN), à desmama (P240), aos 12 (P365) e aos 18 meses (P550) de idade de bovinos da raça Canchim

Table 2 - Means ( $\bar{X})$, standard deviations (S), minimum ( $\mathrm{min}$ ) and maximum values (max), skewness (ske) and kurtosis (kur) for weights at birth (BW), weaning (W240), twelve (W365) and 18 months (W550) of age of Canchim cattle

\begin{tabular}{lrrrrr}
\hline $\begin{array}{l}\text { Característica } \\
\text { Trait }\end{array}$ & $\bar{x}(\mathrm{~kg})$ & $s(\mathrm{~kg})$ & $\min (\mathrm{kg})$ & $\max (\mathrm{kg})$ & $\begin{array}{c}\text { ass } \\
\text { ske }\end{array}$ \\
\hline PN $(B W)$ & 35,22 & 5,50 & 13,00 & 56,00 & 0,2497 \\
P240 (W240) & 198,06 & 35,77 & 53,00 & 340,00 & $-0,0753$ \\
P365 (W365) & 217,93 & 42,68 & 71,00 & 413,00 & 0,2633 \\
P550 (W550) & 282,27 & 50,88 & 119,00 & 545,00 & 0,1026 \\
\hline
\end{tabular}

Tabela 3 - Variâncias (diagonal, $\mathrm{kg}^{2}$ ), covariâncias (acima da diagonal, kg) e correlações (abaixo da diagonal) para os pesos ao nascimento (PN), à desmama (P240), aos 12 (P365) e aos 18 meses (P550) de idade

Table 3 - Variances (diagonal, $\mathrm{kg}^{2}$ ), covariances (above diagonal, $\mathrm{kg}$ ) and correlations (below diagonal) for weights at birth (BW), weaning (W240), twelve (W365) and 18 months (W550) of age of Canchim cattle

\begin{tabular}{|c|c|c|c|c|}
\hline \multirow[b]{2}{*}{$\begin{array}{l}\text { Característica } \\
\text { Trait }\end{array}$} & \multicolumn{4}{|c|}{$\begin{array}{c}\text { Característica } \\
\text { Trait }\end{array}$} \\
\hline & $\mathrm{PN}(B W)$ & $\mathrm{P} 240(W 240)$ & P365 (W365) & P550 (W550) \\
\hline $\mathrm{PN}(B W)$ & 30,23 & 58,80 & 64,76 & 61,34 \\
\hline $\mathrm{P} 240(W 240)$ & 0,30 & $1.279,48$ & $1.168,94$ & $1.169,61$ \\
\hline P365 (W365) & 0,28 & 0,77 & $1.821,87$ & $1.509,44$ \\
\hline P550 (W550) & 0,22 & 0,64 & 0,70 & $2.588,78$ \\
\hline
\end{tabular}

Como demonstrado na Tabela 3 , à medida que o animal fica mais velho, ocorre aumento da variância e, à medida que aumenta o intervalo das pesagens, diminui a correlação entre essas pesagens, fato relatado por Mascioli et al. (1996).

Constam na Tabela 4 os critérios de ajuste proporcionados pelo procedimento MIXED do SAS (2000) para cada arranjo de efeitos fixos, utilizando-se as estruturas de (co)variâncias com homogeneidade e com heterogeneidade de (co)variâncias, e os respectivos graus de liberdade do modelo e do resíduo associados a cada arranjo para os efeitos fixos.

Independentemente da estrutura de (co)variância residual considerada, o arranjo AEF5 proporcionou os menores valores para todos os critérios, sendo, portanto, o de melhor ajuste para a análise dos dados utilizados neste trabalho, enquanto o arranjo AEF2 proporcionou os maiores valores para os critérios, sendo, portanto, o modelo com o pior ajuste para a análise destes dados. Com base nos graus de liberdade (Tabela 4), o arranjo 5 foi o mais completo dos testados, enquanto o arranjo 2 foi o mais simples. Portanto, neste caso, recomenda-se a utilização de modelos mais parametrizados para a análise deste conjunto de dados.
O critério -2RLL (“-2 Residual Log Likelihood”) apontou a estrutura de (co)variância UN como aquela que proporcionou o melhor ajuste para a análise, enquanto o critério CAIC (“Consistent Akaike's Information Criterion”) apontou as estruturas UN e FA(1) como aquelas que proporcionaram os melhores ajustes, exceto para AEF2, em que a estrutura FA(1) foi significativamente melhor que a UN. Essa diferença pode ser atribuída ao fato de o CAIC ser mais rigoroso que o -2RLL por considerar, além do -2RLL, o número de parâmetros a ser estimados e de dados utilizados. A estrutura VC foi a que apresentou os maiores valores para os critérios avaliados e, portanto, foi a pior para representar a variabilidade entre as medidas de cada animal.

A escolha das estruturas FA(1) e UN para representar a variação dentro do indivíduo corrobora os resultados de Freitas et al. (2005), que, estudando dados de nove medidas de pesos de zebuínos, observaram que as estruturas FA(1) e UN foram as mais adequadas para o estudo de medidas repetidas de peso nas raças Gir e Indubrasil. Esses autores também observaram que as estruturas $\mathrm{CSH}$ e $\operatorname{ARMA}(1,1)$ foram adequadas para as raças Guzerá e Nelore, respectivamente.

$\mathrm{Na}$ Tabela 5 são apresentadas as matrizes de (co)variâncias e as correlações entre as medidas de cada animal fornecidas pelas estruturas FA(1) e UN. 
Tabela 4 - Critérios de ajuste (-100.000) para cada arranjo para efeitos fixos (AEF) utilizando-se as estruturas com homogeneidade e com heterogeneidade de variâncias e graus de liberdade do modelo e do resíduo associados aos $A E F^{1}$

Table 4 - Evaluation criteria (-100,000) for fixed effects arrays (FEA) using homogeneous and heterogeneous variance structures and model's and residual's degrees of freedom associated to each FEA

\begin{tabular}{lccccc}
\hline Critério $^{3}$ & AEF1 & AEF3 & AEF4 & AEF5 \\
Criteria $^{3}$ & FEA $1^{2}$ & AEF2 & AEF6 & FEA4 & FEA3 \\
\hline
\end{tabular}

\begin{tabular}{|c|c|c|c|c|c|c|}
\hline \multirow[b]{2}{*}{-2 RLL } & \multicolumn{6}{|c|}{ Auto-regressiva de primeira ordem - $\mathrm{AR}(1)$ (First-order autoregressive) } \\
\hline & $41.676^{\mathrm{eH}}$ & $41.796^{\mathrm{fl}}$ & $40.656^{\mathrm{cI}}$ & $41.199^{\mathrm{dI}}$ & $39.687^{\mathrm{aI}}$ & $40.548^{\mathrm{bI}}$ \\
\hline CAIC & $41.694^{\mathrm{eG}}$ & $41.814^{\mathrm{fH}}$ & $40.674^{\mathrm{cG}}$ & $41.217^{\mathrm{dG}}$ & $39.706^{\mathrm{aG}}$ & $40.566^{\mathrm{bH}}$ \\
\hline
\end{tabular}

Auto-regressiva de primeira ordem com média móvel - $\operatorname{ARMA}(1,1)$ (First-order autoregressive moving average)

\begin{tabular}{|c|c|c|c|c|c|c|}
\hline$-2 R L L$ & $41.699^{\mathrm{eI}}$ & $41.790^{\mathrm{fH}}$ & $40.646^{\mathrm{cH}}$ & $41.190^{\mathrm{dH}}$ & $39.675^{\mathrm{aH}}$ & $40.505^{\mathrm{bH}}$ \\
\hline CAIC & $41.727^{\mathrm{eH}}$ & $41.817^{\mathrm{fH}}$ & $40.674^{\mathrm{cG}}$ & $41.217^{\mathrm{dG}}$ & $39.703^{\mathrm{aG}}$ & $40.533^{\mathrm{bG}}$ \\
\hline
\end{tabular}

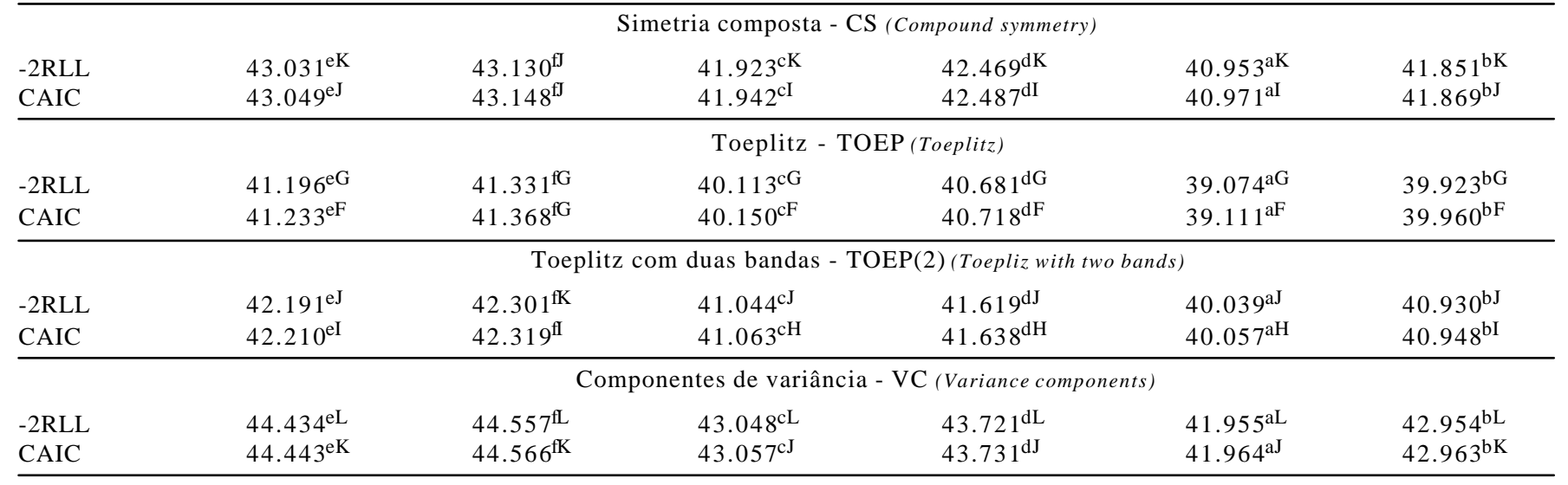

Auto-regressiva heterogênea de primeira ordem - ARH(1)(Heterogeneous first-order autoregressive)

\begin{tabular}{lcccccc}
-2RLL & $32.029^{\mathrm{dD}}$ & $32.141^{\mathrm{eD}}$ & $31.420^{\mathrm{bD}}$ & $31.792^{\mathrm{cD}}$ & $30.846^{\mathrm{aD}}$ & $31.432^{\mathrm{bD}}$ \\
CAIC & $32.075^{\mathrm{dC}}$ & $32.187^{\mathrm{eD}}$ & $31.466^{\mathrm{bC}}$ & $31.838^{\mathrm{cC}}$ & $30.892^{\mathrm{aC}}$ & $31.478^{\mathrm{bC}}$ \\
\hline & & Simetria composta heterogênea $-\mathrm{CSH}$ (Heterogeneous compound symmmetry) \\
-2RLL & $32.571^{\mathrm{dF}}$ & $32.645^{\mathrm{eF}}$ & $31.890^{\mathrm{bE}}$ & $32.262^{\mathrm{cE}}$ & $31.281^{\mathrm{aE}}$ \\
CAIC & $32.617^{\mathrm{dE}}$ & $32.691^{\mathrm{eF}}$ & $31.936^{\mathrm{bD}}$ & $32.308^{\mathrm{cD}}$ & $31.327^{\mathrm{aD}}$ & $31.878^{\mathrm{bE}}$ \\
\hline
\end{tabular}

Fator analítico de primeira ordem - FA(1) (First-order factor analytic)

\begin{tabular}{lcccccc}
-2RLL & $30.911^{\mathrm{dB}}$ & $30.939^{\mathrm{eB}}$ & $30.386^{\mathrm{bB}}$ & $30.694^{\mathrm{cB}}$ & $29.869^{\mathrm{aB}}$ & $30.384^{\mathrm{bB}}$ \\
CAIC & $30.984^{\mathrm{dA}}$ & $31.012^{\mathrm{eA}}$ & $30.460^{\mathrm{bA}}$ & $30.768^{\mathrm{cA}}$ & $29.942^{\mathrm{aA}}$ & $30.457^{\mathrm{bA}}$ \\
\hline & & Huynh - Feldt - HF (Huynh - Feldt) & \\
-2RLL & $32.484^{\mathrm{dE}}$ & $32.492^{\mathrm{dE}}$ & $31.961^{\mathrm{bF}}$ & $32.271^{\mathrm{cF}}$ & $31.440^{\mathrm{aF}}$ \\
CAIC & $32.530^{\mathrm{dD}}$ & $32.538^{\mathrm{dE}}$ & $32.007^{\mathrm{bE}}$ & $32.317^{\mathrm{cE}}$ & $31.486^{\mathrm{aE}}$ & $31.958^{\mathrm{bF}}$ \\
\hline
\end{tabular}

\begin{tabular}{llcccc}
\hline \multicolumn{5}{c}{ Toeplitz heterogênea - TOEPH (Heterogeneous Toeplitz) } \\
-2RLL & $31.936^{\mathrm{dC}}$ & $32.038^{\mathrm{eC}}$ & $31.317^{\mathrm{bC}}$ & $31.684^{\mathrm{cC}}$ & $30.746^{\mathrm{aC}}$ \\
CAIC & $32.001^{\mathrm{dB}}$ & $32.103^{\mathrm{eC}}$ & $31.382^{\mathrm{bB}}$ & $31.749^{\mathrm{cB}}$ & $31.328^{\mathrm{bC}}$ \\
\hline & & Não-estruturada - UN (Unstructured) & $31.393^{\mathrm{bB}}$ \\
-2RLL & $30.892^{\mathrm{dA}}$ & $30.927^{\mathrm{eA}}$ & $30.369^{\mathrm{bA}}$ & $30.679^{\mathrm{cA}}$ & $29.848^{\mathrm{aA}}$ \\
CAIC & $30.984^{\mathrm{dA}}$ & $31.019^{\mathrm{eB}}$ & $30.461^{\mathrm{bA}}$ & $30.772^{\mathrm{cA}}$ & $29.940^{\mathrm{aA}}$ \\
\hline
\end{tabular}

\begin{tabular}{lrrrrr} 
Modelo (Model) & 59 & 51 & 176 & 106 & 293 \\
Resíduo (Residual) & 14.700 & 17.708 & 14.583 & 14.653 & 14.466 \\
\hline
\end{tabular}

${ }^{1}$ Letras minúsculas diferentes na mesma linha e maiúsculas diferentes na mesma coluna representam diferença significativa $(P<0,05)$ pelo teste de ${ }^{2}$.

${ }^{2} \mathrm{AEF} 1$ = efeitos principais (EPs) de ano e mês de nascimento e sexo do bezerro; AEF2 = EPs de ano e trimestre de nascimento e sexo do bezerro; AEF3 = grupo de contemporâneos (GCs) composto por ano e mês de nascimento e EP de sexo do bezerro; AEF4 = GCs composto por ano e trimestre de nascimento e EP de sexo do bezerro; AEF5 = GCs composto por ano, mês de nascimento e sexo do bezerro; AEF6 = GCs composto por ano, trimestre de nascimento e sexo do bezerro.

3 -2RLL = "-2 Residual Log Likelihood" e CAIC = "Consistent Akaike's Information Criterion".

1 Different small letters in the same row indicate statistical difference $(P<.05)$ by $\chi^{2}$ test. Different capital letters in the same column indicate statistical difference $(P<.05)$ by $?^{2}$ test.

2 FEA1 = main effects (MES) of year and month of birth and sex of calf; FAE2 = ME of year and season of birth and sex of calf; FEA3 = contemporary group (CG) of year and month of birth and $M E$ of sex of calf; $F E A 4=C G$ of year and season of birth and sex of calf; $F E A 5=C G$ of year and month of birth and sex of calf; $F E A G=C G$ of year and season of birth and sex of calf.

$3-2 R L L=-2$ Residual Log Likelihood and CAIC = Consistent Akaike's Information Criterion. 
Tabela 5 - Variâncias (diagonal, $\mathrm{kg}^{2}$ ), covariâncias (acima da diagonal, kg) e correlações (abaixo da diagonal) para os pesos ao nascimento (PN), à desmama (P240), aos 12 (P365) e aos 18 meses (P550) de idade fornecidas pelas estruturas Fator Analítico de Primeira Ordem $(F A(1))$ e Não-estruturada (UN), considerando o modelo com o grupo de contemporâneos de ano, mês de nascimento e sexo do bezerro (AEF5)

Table 5 - Variances (diagonal, $\mathrm{kg}^{2}$ ), covariances (above diagonal, $\mathrm{kg}^{2}$ ) and correlations (below diagonal) of weights at birth (BW), weaning (W240), twelve (W365) and eighteen months (W550) of age from First-Order Factor Analytic ( $F A(1))$ and Unstructured (UN) for the model including the contemporary group of year and month of birth and sex of calf (FEA5)

\begin{tabular}{lrrrr}
\hline & PN & P240 & P365 & P550 \\
& $B W$ & $W 240$ & $W 365$ & $W 550$ \\
\hline \multicolumn{5}{c}{$\mathrm{FA}(1)$} \\
\hline PN $(B W)$ & 27,10 & 49,08 & 62,97 & 63,44 \\
P240 (W240) & 0,26 & $1.270,96$ & $1.163,11$ & $1.171,77$ \\
P365 (W365) & 0,28 & 0,77 & $1.817,50$ & $1.503,47$ \\
P550(W550) & 0,23 & 0,65 & 0,69 & $2.590,64$ \\
\hline \multicolumn{5}{c}{$\mathrm{UN}$} \\
\hline PN (BW) & 27,00 & 55,65 \\
P240 (W240) & 0,30 & $1.276,41$ & $1.158,37$ & $1.164,95$ \\
P365 (W365) & 0,25 & 0,76 & $1.803,79$ & $1.497,27$ \\
P550 (W550) & 0,21 & 0,64 & 0,69 & $2.582,52$ \\
\hline
\end{tabular}

Tabela 6 - Coeficientes de assimetria e curtose, média e mediana dos resíduos para os pesos ao nascimento (PN), à desmama (P240), aos 12 (P365) e aos 18 meses (P550) de idade, obtidos das análises utilizando-se as estruturas de (co)variância residual Fator Analítico de Primeira Ordem (FA(1)) e Não-Estruturada (UN) e das análises unicaracterísticas, considerando-se o modelo com o efeito do grupo de contemporâneos de ano, mês de nascimento e sexo do bezerro (AEF5)

Table 6 - Skewness and kurtosis coefficients, mean and median of residuals for weights at birth (BW), weaning (W240), twelve (W365) and eighteen months (W550) of age using the FirstOrder Factor Analytic (FA(1)) and Unstructured (UN) obtained by single trait models including the effect of contemporary group of year and month of birth and sex of calf (FEA5)

\begin{tabular}{lcccc}
\hline $\begin{array}{l}\text { Característica } \\
\text { Trait }\end{array}$ & $\begin{array}{c}\text { Assimetria } \\
\text { Skewness }\end{array}$ & $\begin{array}{c}\text { Curtose } \\
\text { Kurtosis }\end{array}$ & $\begin{array}{c}\text { Média } \\
\text { Mean }\end{array}$ & $\begin{array}{c}\text { Mediana } \\
\text { Median }\end{array}$ \\
\hline \multicolumn{5}{c}{ FA(1) } \\
\hline PN $(B W)$ & 0,2188 & 0,3232 & 0 & $-0,1514$ \\
P240 $(W 240)$ & $-0,0754$ & 0,0744 & 0 & 0,7712 \\
P365 (W365) & 0,3129 & 0,4123 & 0 & $-1,8851$ \\
P550 (W550) & 0,2229 & 0,4701 & 0 & $-0,8307$ \\
\hline \multicolumn{5}{c}{$\mathrm{UN}$} \\
\hline PN $(B W)$ & 0,2286 & 0,3439 & 0 & $-0,1764$ \\
P240 (W240) & $-0,0792$ & 0,0689 & 0 & 0,7982 \\
P365 (W365) & 0,3080 & 0,4103 & 0 & $-1,8597$ \\
P550 (W550) & 0,2194 & 0,4720 & 0 & $-0,6750$ \\
\hline & Análises unicaracterísticas $($ Singletrait analyses $)$ \\
\hline PN $(B W)$ & 0,2061 & 0,3149 & 0 & $-0,1552$ \\
P240 $(W 240)$ & $-0,2815$ & 1,3844 & 0 & 0,2537 \\
P365 (W365) & $-0,1165$ & 1,3884 & 0 & 0,8240 \\
P550 (W550) & $-0,0222$ & 1,4935 & 0 & 0,8040 \\
\hline
\end{tabular}

As estruturas FA(1) e UN fornecem matrizes em que é possível observar aumento da variância ao longo da vida do animal, fenômeno reportado por Mascioli et al. (1996) e Freitas et al. (2005). Além disso, fornecem correlações diferentes para cada par de idades, que, geralmente, são menores à medida que aumenta o intervalo de pesagens. A escolha das estruturas FA(1) e UN para modelar as (co)variâncias entre as medidas de peso do mesmo animal é coerente com o fenômeno analisado e, portanto, corrobora a aplicação dos resultados obtidos neste trabalho.

$\mathrm{Na}$ Tabela 6 são apresentados os coeficientes de assimetria e de curtose, a média e a mediana dos resíduos para PN, P240, P365 e P550, obtidos das análises com as estruturas de (co)variâncias residuais Fator Analítico de Primeira Ordem e Não-Estruturada e das análises de variância unicaracterísticas, considerando-se o modelo com o efeito do grupo de contemporâneos de ano, mês de nascimento e sexo do bezerro (AEF5). A moda não é apresentada porque, em todos os casos, o conjunto de resíduos foi multimodal.

Os coeficientes de assimetria dos resíduos obtidos das análises utilizando-se estruturas de (co)variâncias residuais e das análises unicaracterísticas foram semelhantes, porém, observou-se redução dos coeficientes de curtose quando da utilização das estruturas de (co)variâncias em relação àqueles obtidos nas análises unicaracerísticas. Os valores obtidos foram próximos de zero e indicam que a análise de medidas repetidas para estes dados, com as pressuposições iniciais, ainda que não completamente atendidas, podem gerar resultados confiáveis.

Dessa forma, o estudo do peso dos animais como medidas repetidas pode ser mais eficiente que o dos pesos em cada idade como características diferentes, pois a utilização de estruturas de (co)variâncias residuais possibilita considerar as oscilações da variância e a correlação entre os resíduos, implicando estimação de parâmetros genéticos e/ou ambientais mais adequados e realização de testes de hipóteses mais confiáveis.

\section{Conclusões}

$\mathrm{O}$ arranjo mais adequado dos efeitos fixos para representar a variabilidade dos pesos dos animais dentro do rebanho foi o grupo de contemporâneos formado por ano, mês e sexo do bezerro.

As melhores estruturas de (co)variâncias residuais foram a Fator Analítico de Primeira Ordem e a NãoEstruturada, que consideram aumento das variâncias com o aumento da idade do indivíduo e correlações diferentes para cada par de medidas de peso. 


\section{Literatura Citada}

ABCCAN-Embrapa-Geneplus. Sumário de touros Canchim e MA. Edição Primavera/2002. Campo Grande: Geneplus Consultoria Agropecuária LTDA, 2002. Disponível em: http: <www.canchim.com.br/dep.htm\#item7>. Acesso em: $15 / 06 / 2003$

BOZDOGAN, H. Model selection and Akaike's information criterion (AIC): the general theory and its analytical extensions. Psychometrika, v.52, n.3, p.345-370, 1987.

FREITAS, A.R. Técnicas de análises exploratórias de dados. In: REUNIÃO ANUAL DA SOCIEDADE BRASILEIRA DE ZOOTECNIA, 40., 2003, Santa Maria. Anais... Santa Maria: SBZ/Infovia, 2003. (CD-ROM)

FREITAS, A.R.; PRESOTTI, C.V.; TORAL, F.L.B. Alternativas de análises em dados de medidas repetidas de bovinos de corte. Revista Brasileira de Zootecnia, v.34, n.6, p.2233-2244, 2005 (supl.).

GRÖHN, Y.T.; McDERMOTT, J.J.; SCHUKKEN, Y.H. et al. Analysis of correlated continuous repeated observations: modeling the effect of ketosis on milk yield in dairy cows. Preventive Veterinary Medicine, v.39, n.2, p.137-153, 1999.

LAIRD, N.M.; WARE, J.H. Random effects models for longitudinal data. Biometrics, v.38, n.4, p.963-974, 1982.

LORENZO-BERMEJO, J.; ROEHE, R.; RAVE, G. et al. Comparison of linear and nonlinear functions and covariance structures to estimate feed intake pattern in growing pigs. Livestock Production Science, v.82, n.1, p.15-26, 2003.
LITTELL, R.C.; HENRY, P.R.; AMMERMAN, C.B. Statistical analysis of repeated measures data using SAS procedures Journal of Animal Science, v.76, n.4, p.1216-1231, 1998.

MASCIOLI, A.S.; ALENCAR, M.M.; BARBOSA, P.F. et al. Influência de fatores de meio sobre pesos de animais da raça Canchim. Revista da Sociedade Brasileira de Zootecnia, v.25, n.5, p.853-865, 1996.

MELLO, S.P.; ALENCAR M.M.; SILVA, L.O.C. et al. Estimativas de (co)variâncias e tendências genéticas para pesos em um rebanho Canchim. Revista Brasileira de Zootecnia, v.31, n. 4, p. 1707-1714, 2002 .

STATISTICAL ANALYSIS SYSTEM - SAS. User's guide Version 8. 2.ed. Cary: 2000. (CD-ROM)

VONESH, E.F.; CHINCHILLI, V.M.; PU, K. Goodness of fit in generalized nonlinear mixed effects model. Biometrics, v.52, n.2, p.572-587, 1996.

WOLFINGER, R. Covariance structure selection in general mixed models. Community of Statistics - Simulation, v.22, n.4, p.1079-1106, 1993.

Recebido: 17/06/04 Aprovado: 29/05/06 